\title{
In Frail Elderly Patients, Low-Dose Gemcitabine over 6-Hour Infusion Is Equally Effective and Less Toxic Than the Standard Gemcitabine Protocol for Advanced Pancreatic Adenocarcinoma: A Randomized Phase II Trial
} \author{
Anwar Tawfik Amin'3 , Muhammad Abbas El-Masry", Mona M. Sayed ${ }^{5}$, Dalia Osama ${ }^{5}$ \\ ${ }^{1}$ Medical Oncology Department, South Egypt Cancer Institute, Assiut University, Assiut, Egypt \\ ${ }^{2}$ Clinical Oncology and Nuclear Medicine Department, Sohag University, Sohag, Egypt \\ ${ }^{3}$ Surgical Oncology Department, South Egypt Cancer Institute, Assiut University, Assiut, Egypt \\ ${ }^{4}$ Division of gastroenterology-Department of internal medicine, Assiut University, Assiut, Egypt \\ ${ }^{5}$ Radiation Oncology Department, South Egypt Cancer Institute, Assiut University, Assiut, Egypt \\ Email: *salahmab76@yahoo.com
}

Salah Mabrouk Khallaf ${ }^{*}$, Elsayed Mostafa Ali2, Ahmed M. Maklad2, Mohamed Medhat Samir²,

How to cite this paper: Khallaf, S.M., Ali, E.M., Maklad, A.M., Samir, M.M., Amin, A.T., El-Masry, M.A., Sayed, M.M. and Osama, D. (2020) In Frail Elderly Patients, Low-Dose Gemcitabine over 6-Hour Infusion Is Equally Effective and Less Toxic Than the Standard Gemcitabine Protocol for Advanced Pancreatic Adenocarcinoma: A Randomized Phase II Trial. Journal of Cancer Therapy, 11, 124-141.

https://doi.org/10.4236/jct.2020.113011

Received: February 20, 2020

Accepted: March 21, 2020

Published: March 24, 2020

Copyright $\odot 2020$ by author(s) and Scientific Research Publishing Inc. This work is licensed under the Creative Commons Attribution International License (CC BY 4.0).

http://creativecommons.org/licenses/by/4.0/ (c) (i) Open Access

\section{Abstract}

Background: Treatment of frail elderly patients with pancreatic cancer is still a major problem due to intolerance to standard chemotherapy doses. Aim: This study aims to compare the low-dose gemcitabine over 6 hours (LD6H) to the standard gemcitabine protocol in terms of clinical benefit, survival, and safety in the frail elderly patients with advanced pancreatic adenocarcinoma. Methods: Patients enrolled in this trial were randomly assigned by in a 1:1 fashion via closed envelope method to either receive gemcitabine of 1000 $\mathrm{mg} / \mathrm{m}^{2}$ over 30 -minute infusion on days 1,8 , and 15 of every 4-week cycle (standard protocol arm) or gemcitabine as a weekly low-dose $\left(250 \mathrm{mg} / \mathrm{m}^{2}\right)$ over 6-hour infusion (LD6H arm). Results: We enrolled eighty-two eligible frail elderly patients with advanced pancreatic cancer. The patients were randomly assigned to receive either standard gemcitabine protocol (40 patients) or low-dose $\left(250 \mathrm{mg} / \mathrm{m}^{2}\right)$ gemcitabine over 6-hour infusion, given weekly (42 patients). There was no significant difference between the standard group and low-dose group as regard of the overall response rate $(\mathrm{p}=0.654)$, the disease control rate $(D C R)(p=0.845)$, the median progression-free survival (PFS) ( $p$ $=0.908)$ and the overall survival (OS) $(\mathrm{p}=0.331)$. The low-dose regimen had a significantly lower incidence of adverse effects grades 3 or 4 when compared to the standard regimen: $(\mathrm{p}=0.024$ for fatigue, $\mathrm{p}=0.027$ for hypoten- 
sion, $\mathrm{p}=0.012$ for each anemia as well as thrombocytopenia, and $\mathrm{p}=0.006$ for neutropenia). Conclusion: Low-dose gemcitabine over 6-hour infusion is equally effective and less toxic when compared to standard gemcitabine protocol in frail elderly patients with advanced pancreatic adenocarcinoma. So, we recommend the low-dose gemcitabine for frail elderly patients with advanced pancreatic cancer.

\section{Keywords}

Continuous Infusion, Elderly, Frail, Low Dose Gemcitabine, Pancreatic Cancer

\section{Introduction}

Pancreatic cancer is one of the major worldwide health problems as it is the 11th most common cancer in the world [1]. It has the worst overall survival among all cancers ranking as the seventh most common cause of death from cancer worldwide [1]. Worldwide incidence and mortality of pancreatic cancer correlate with increasing age [1]. Most cases are diagnosed in the advanced stage as it has spread when a patient complains of tumor-specific symptom [2].

A landmark randomized phase III study conducted by Burris et al. comparing gemcitabine versus 5-fluorouracil in the treatment of advanced pancreatic cancer. They confirmed a statistically significant survival advantage favoring gemcitabine arm in terms of median and 1-year overall survival (5.7 months and $18 \%$ vs. 4.4 months and $2 \%$, respectively; $\mathrm{P}=0.0025$ ) [3]. Gemcitabine became the standard regimen in patients with advanced or metastatic disease based on the previous study [3].

PRODIGE 4/ACCORD 11 Phase III trial stated the superiority of FOLFIRINOX regimen compared to gemcitabine as first-line therapy for metastatic pancreatic cancer as regard of overall survival (11.1 months vs 6.8 months for FOLFIRINOX and gemcitabine, respectively; $\mathrm{HR}=0.57, \mathrm{P}<0.001$ ) [4]. Due to the high toxicity of FOLFIRINOX, they exclude patients with performance status 2 or elderly patients (age more than 65 years) [4].

Another phase III clinical trial "MPACT trial" reported that the combination of gemcitabine and nab-paclitaxel was more effective than gemcitabine monotherapy for metastatic pancreatic cancer [5]. A recent review article published by Macchini et al. (2019) [6] that mentioned the limitation of the MPACT study in respect of age, and they said: "This MPACT results reported overall acceptable toxicity even if, once more, data were not stratified by age".

Treatment of the frail elderly patients is still challenging. "Frailty" is the accumulation of multiple physical and psychosocial deficits in the older person [7]. A gait speed $<0.8 \mathrm{~m} / \mathrm{s}$ (taking $>5$ seconds to walk 4 meters) or a timed-upand-go-test (TUGT) $>10$ seconds plus a score of $\geq 3$ on the PRISMA 7 (Program of Research to Integrate the Services for the Maintenance of Autonomy 7) score 
indicates the frailty [8] [9]. Frail elderly people have diminished the capacity to compensate for stressors compared to people of the same chronological age, implying a state of elevated risk in the context of treatment decision-making [10]. Elderly patients are defined by the World Health Organization (WHO) as people older than 65 years. [11]

Gemcitabine has antitumor activity in elderly patients with locally advanced and metastatic pancreatic ductal adenocarcinoma in several studies [12] [13]. Also, in a review article, Higuera et al. recommended gemcitabine monotherapy for frail elderly patients [14].

Many Phase I studies were constructed trying to minimize the gemcitabine toxicity without decreasing its efficacy. These phase I studies [15] [16] [17] stated that the prolonged infusion of gemcitabine during 3-, 4-, 6-, and 24-hour at low dose levels in patients with advanced solid tumors were safe. Also, in these studies, the doses value between 180 and $450 \mathrm{mg} / \mathrm{m}^{2}$. The aim of our phase II trial was to compare the low dose gemcitabine over 6 hours to the standard gemcitabine protocol in terms of clinical benefit and survivals in the frail elderly patients with advanced pancreatic adenocarcinoma.

\section{Methods}

\section{Sample Size}

We started our trial without calculation of the sample size. However, we assessed all available patients (112 patients) for eligibility criteria during the study period (40 months), we assumed that is enough number for analysis based on that our sample size is much more than that of previous phase II randomized trial published by Sakamoto et al. who enrolled 25 patients and analysed 21 eligible patients.

\section{Patient Selection}

The eligibility criteria were the evidence of the advanced pancreatic cancer, both sexes, age of 65 years or older, frail patients (A gait speed $<0.8 \mathrm{~m} / \mathrm{s}$ [taking $>5 \mathrm{~s}$ to walk $4 \mathrm{~m}$ ] or a timed-up-and-go-test (TUGT test) $>10$ seconds with a score of $\geq 3$ on the PRISMA 7 score) [8] [9], an Eastern Cooperative Oncology Group (ECOG) performance status 2, patients not receiving prior chemotherapy, and adequate hematologic, hepatic, and renal functions. Relieving of obstructive jaundice either by stenting via endoscopic retrograde cholangiopancreatography (ERCP) or surgically by choledochojejunostomy or hepaticojejunostomy was allowed. Also, palliative radiotherapy for distant metastasis was allowed if indicated. Exclusions criteria were the evidence of second primary malignancy, concurrent local radiotherapy, and any psychiatric disease or social problem that would affect the compliance of participants. Also, patients with unknown tumor responses were excluded.

During the study period from May 2016 to September 2019, we assessed 112 patients for eligibility. Our manuscript reporting adheres to CONSORT guidelines for reporting clinical trials (CONSORT diagram: Appendix I, CONSORT checklist: Appendix II). 
Our trial was approved by the ethics committee and institutional review board under the number of SECI-IRB-IORG0006563: No: 254 on 28 March 2016. The committee that approved the research confirmed that all research was performed in accordance with relevant guidelines/regulations. The informed consent was obtained from all participants and/or their legal guardians. The drugs were supplied by governmental and health insurance at the location of the study.

\section{Study Design and Treatment Plan}

Patients enrolled in this trial were randomly assigned by the corresponding author in a 1:1 fashion via closed envelope method to either receive gemcitabine of $1000 \mathrm{mg} / \mathrm{m}^{2}$ over 30 -minute infusion on days 1,8 , and 15 of every 4 -week cycle (standard protocol arm) or weekly low-dose $\left(250 \mathrm{mg} / \mathrm{m}^{2}\right)$ over 6-hour infusion (LD6H arm). The gemcitabine dose was reduced to $75 \%$ of the original cycle initiation dose, in the case of the following hematological toxicities: absolute granulocyte counts less than $500 \times 10^{6} / \mathrm{L}$ for more than 5 days, febrile neutropenia, platelets less than $25 \times 10^{9} / \mathrm{L}$, or cycle delay of more than 1 week due to toxicity. Patients were categorized according to age (75 years is cutoff between the 2 age groups), gender (male vs. female), PRIMSA 7 score (score $3 / 4$ vs. score 5 - 7), tumor grade ( $1 / 2$ vs. 3 ), tumor location (pancreatic head, body, tail, or diffuse), liver metastasis (yes vs. no), lung metastasis (yes vs. no), and the number of metastatic organ(s) (0/1 vs. two or more). The crossover between the two arms was not allowed. Patients were continued to receive either regimen until disease progression, unacceptable toxicity, study end, or on patient demand.

\section{Assessments}

Baseline evaluation included a detailed history, physical examination, hematological counts, renal, and hepatic functions tests. Assessment of performance status was done based on the ECOG scale. Assessment of frailty via gait speed, TGUT test and PRIMSA 7 score (Appendix III). Radiographic tumor assessments by computed tomography or magnetic resonance imaging were performed at baseline, every 8 weeks, and in the presence of any suspicion of tumor progression. The response was assessed by the investigators according to the Response Evaluation Criteria in Solid Tumors (RECIST 1.1) [18]. At each gemcitabine administration, all adverse events were assessed according to the Common Terminology Criteria for Adverse Events (CTCAE 4.03) [19].

\section{Statistical Analysis}

The primary end-points were progression-free survival (PFS), defined as the time from the start of treatment to disease progression or death from any cause, whichever came first, and safety through assessment toxicity profile based on CTCAE 4.03. Secondary end-points were overall survival (OS; defined as the time from the start of treatment to date of death from any cause, or date of last follow up, whichever came first.), overall response rate (ORR; defined as sum of rates of complete response (CR) and partial response (PR) to chemotherapy), and disease control rate (DCR; defined as sum of rates of stable disease (SD), complete response, and partial response). Univariate analysis was used through the presentation of continuous variables as median and range. Categorical varia- 
bles are presented as frequency and percentage. Bivariate analysis was done to compare categorical variables using Chi-Square test or Fisher Exact test when appropriate. Kaplan-Meier method was used to estimate the survival time distribution and the median survival of each treatment group. The treatment difference between the two groups was assessed by a log-rank test. Hazard Ratios (HRs) and 95\% confidence intervals (CIs) were determined by using a cox proportional hazards model. Factors were re-assessed by multivariate analysis by using Cox regression analysis. Median follow up time for all patients was derived from the reverse Kaplan-Meier method. A P-value less than 0.05 is considered as a cut off of significance. SPSS version 21 (SPSS Inc. Chicago, IL, USA) was used in the storage and analysis of data [20].

\section{Results}

\section{Patients' Chart}

During the study period from May 2016 to September 2019, we assessed 112 patients for eligibility. Ten patients were excluded from standard arm due to death before starting treatment $(n=4)$, lost follow up after $1^{\text {st }}$ cycle without assessment $(n=3)$, and patient demand $(n=3)$; while eight patients from the experimental arm were excluded from analysis, five of them died before first assessment and the remaining three patients withdrew their consent. The last day of the follow up was 30 September 2019 as the study was completed and prepared for analysis. Eighty-two patients were analyzed for study endpoints; 40 patients in standard arm and 42 patients in LD6H arm. CONSORT diagram shows the patients' chart (Appendix I).

\section{Demographics and Patients' Characteristics}

The data is shown in Table 1. The median ages were 70.5 and 69.5 years for the standard arm and LD6H arm respectively, with a range of $65-81$ years for each. The gender was fairly distributed within the two arms $(55 \%$ males in standard arm and $52.4 \%$ in LD6H arm). The metastases affected only one organ in 19 cases $(47.5 \%)$ in the standard arm vs. 24 cases $(57.1 \%)$ in the LD6H arm. The liver was the most common site of distant metastasis (75\% for standard arm and $69 \%$ for LD6H arm).

\section{Efficacy}

\section{Response Rates}

The partial response rate was $17.5 \%$ in the standard arm and $21.4 \%$ in the LD6H arm. One-quarter (25\%) of patients receiving the standard protocol remained stable versus $21.4 \%$ in the $\mathrm{LD} 6 \mathrm{H}$ arm. There was no case of complete response (CR) in both arms. There was no significant difference between the standard group and LD6H group as regard ORR and DCR (17.5\% vs. $21.4 \%$ respectively; $\mathrm{p}=0.654$ for ORR and $45 \%$ vs. $42.9 \%$ respectively; $\mathrm{p}=845)$ (Table 2 ).

\section{Survival End-Points}

Progression-Free Survival (PFS)

After a median follow-up of 9 months, the median PFS was 5 months in both groups, with 95\% CI, 2.78 to 7.22 for standard group and 95\% CI, 3.89 to 6.12 
Table 1. Demographics and baseline characteristics of enrolled patients $(n=82)$ received standard gemcitabine protocol or gemcitabine of low dose over 6-hour infusion.

\begin{tabular}{|c|c|c|c|c|}
\hline \multirow{2}{*}{ Characteristic } & \multicolumn{2}{|c|}{ Standard Gemcitabine Protocol $(n=40)$} & \multicolumn{2}{|c|}{ Gemcitabine LD6H $(\mathrm{n}=42)$} \\
\hline & No & $\%$ & No & $\%$ \\
\hline \multicolumn{5}{|c|}{ Age, Years } \\
\hline Median (Range) & \multicolumn{2}{|c|}{$70.5(65-81)$} & \multicolumn{2}{|c|}{$69.5(65-81)$} \\
\hline$<75$ & 29 & 72.5 & 32 & 76.2 \\
\hline$\geq 75$ & 11 & 27.5 & 10 & 23.8 \\
\hline \multicolumn{5}{|c|}{ Sex } \\
\hline Male & 22 & 55 & 22 & 52.4 \\
\hline Female & 18 & 45 & 20 & 47.6 \\
\hline \multicolumn{5}{|c|}{ PRIMSA 7 Score } \\
\hline Score $3-4$ & 18 & 45 & 20 & 47.5 \\
\hline Score 5 - 7 & 22 & 55 & 22 & 52.4 \\
\hline \multicolumn{5}{|c|}{ Pancreatic Tumor Site } \\
\hline Head & 21 & 52.5 & 26 & 61.9 \\
\hline Body & 9 & 22.5 & 8 & 19 \\
\hline Tail & 6 & 15 & 7 & 16.7 \\
\hline Multicentric & 4 & 10 & 1 & 2.4 \\
\hline \multicolumn{5}{|c|}{ Tumor Grade } \\
\hline Grade I/II & 25 & 62.5 & 24 & 57.1 \\
\hline Grade III & 15 & 37.5 & 18 & 42.9 \\
\hline \multicolumn{5}{|l|}{ Liver Metastasis } \\
\hline Yes & 30 & 75 & 29 & 69 \\
\hline No & 10 & 25 & 13 & 31 \\
\hline \multicolumn{5}{|l|}{ Lung Metastasis } \\
\hline Yes & 10 & 25 & 15 & 35.7 \\
\hline No & 30 & 75 & 27 & 64.3 \\
\hline \multicolumn{5}{|c|}{ No. of Metastasized Organ (s) } \\
\hline One Organ & 19 & 47.5 & 24 & 57.1 \\
\hline Two or More & 21 & 52.5 & 18 & 42.9 \\
\hline \multicolumn{5}{|c|}{ Chemotherapy Cycles } \\
\hline Total Number & \multicolumn{2}{|c|}{205} & \multicolumn{2}{|c|}{226} \\
\hline Mode & \multicolumn{2}{|c|}{6} & \multicolumn{2}{|c|}{3} \\
\hline Range & \multicolumn{2}{|c|}{$2-12$} & \multicolumn{2}{|c|}{$2-17$} \\
\hline
\end{tabular}

Abbreviations: ECOG PS, Eastern Cooperative Oncology Group performance status; LD6H, low dose over 6 hours; PRIMSA 7, Program of Research to Integrate the Services for the Maintenance of Autonomy. ${ }^{*}$ The grade was not known because the tissue diagnosis couldn't be done. 
Table 2. Response rates according to recist criteria in patients received standard gemcitabine protocol or gemcitabine of low dose over 6-hour infusion.

\begin{tabular}{ccccc}
\hline & \multicolumn{2}{c}{$\begin{array}{c}\text { Standard Gemcitabine Protocol } \\
(\mathbf{n}=40)\end{array}$} & \multicolumn{2}{c}{$\begin{array}{c}\text { Gemcitabine LD6H } \\
(\mathbf{n}=42)\end{array}$} \\
\hline Characteristic & No & $\%$ & No & $\%$ \\
\hline CR & 0 & 0 & 0 & 0 \\
PR & 7 & 17.5 & 9 & 21.4 \\
SD & 10 & 25 & 9 & 21.4 \\
PD & 23 & 57.5 & 24 & 57.1 \\
ORR (CR + PR) & 7 & 17.5 & 9 & 21.4 \\
CHI-SQUARE P-VALUE & & $\mathrm{P}=0.654$ & & \\
DCR (CR + PR + SD) & 18 & 45 & 18 & 42.9 \\
CHI-SQUARE P-VALUE & & $\mathrm{P}=0.845$ & & \\
\hline
\end{tabular}

Abbreviations: CR, complete response; DCR, disease control rate; LD6H, low dose over 6 hours; ORR, overall response rate; $\mathrm{PR}$, partial response; RECIST, response evaluation criteria in solid tumors; SD, stable disease.

for LD6H group; log-rank $\mathrm{p}=0.908$; unadjusted HR 1.07; $95 \%$ CI 0.60 to 1.90 (Figure 1(a)). Adjusted HR through multivariate analysis confirmed the lack of significant effect of chemotherapy type on PFS (adjusted HR 1.08; 95\% CI 0.65 to $1.7 ; \mathrm{p}=0.762$ ) (attached complementary file 1 ). Subgroup analysis within the study arms revealed the absence of any significant effect of any subgroup of the examined parameter on PFS (Figure 2(a)).

Overall Survival (OS)

Median OS was not significantly different between the two arms: 10 months; 95\% CI 8.95 to 11.05 in the standard protocol arm versus 8 months; 95\% CI 6.41 to 11.59 in $\mathrm{LD} 6 \mathrm{H}$ arm; unadjusted HR $1.25,95 \%$ CI 76 to 2.05 ; log-rank $\mathrm{p}=$ 0.331; Figure 1(b). Regarding OS, there was an absent of any significant interaction between the studied factors including chemotherapy type. The adjusted HR for chemotherapy type was $1.45 ; 95 \%$ CI $.85-2.46 ; \mathrm{p}=0.170$ (complementary file 2). Also, no subgroup of the examined parameters had a significant effect on OS as shown in the forest plot Figure, titled Figure 2(b).

\section{Adverse Events}

Totally, patients in standard protocol arm received a number of 205 cycles of treatment (mode, 6; range 2 - 12) versus 226 cycles (mode 3 , range 2 - 17) in the LD6H group (Table 1). Most hematologic and nonhematologic adverse events were grade 1 or 2 intensity (Table 3 ). For all grades, fatigue was the most common nonhematologic adverse effect in both groups (37.5\% in standard protocol group and $31 \%$ in LD6H group) followed by anorexia (30\%) in standard protocol group and hypotension (17.5\%) in LD6H group. The thrombocytopenia was the most frequently reported hematologic side effect in standard protocol and LD6H arms (45\% and $38.1 \%$ respectively) (Table 3 ). 


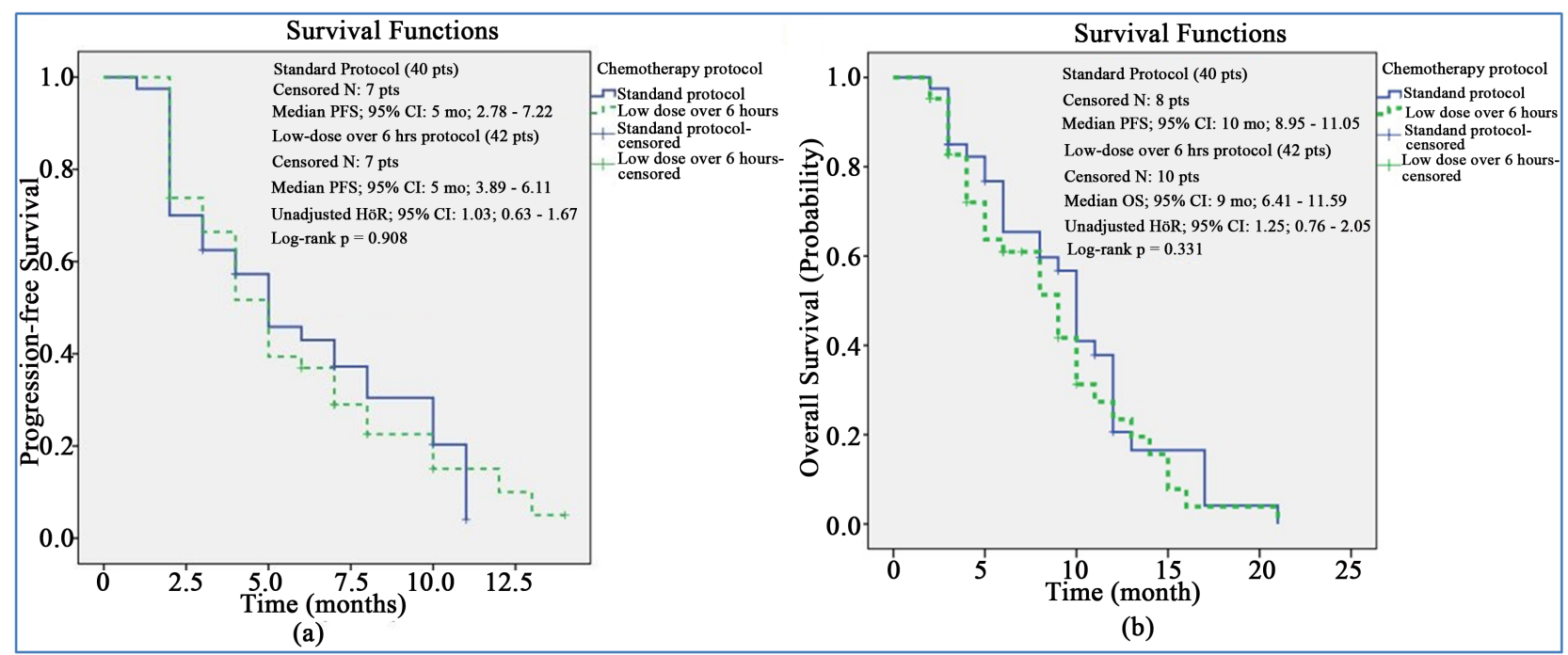

Figure 1. Kaplan-Meier plot for (a) PFS and (b) OS. CI: confidence interval; HR: hazard ratio; OS: overall survival; PFS: progression-free survival.

Table 3. Toxicities according to CTCAE (version 4.03) in patients received standard gemcitabine protocol or gemcitabine of low dose over 6-hour infusion.

\begin{tabular}{|c|c|c|c|c|c|}
\hline \multirow{2}{*}{ EVENT } & \multicolumn{2}{|c|}{$\begin{array}{l}\text { Standard Gemcitabine Protocol } \\
\qquad(\mathrm{n}=40)\end{array}$} & \multicolumn{2}{|c|}{$\begin{array}{l}\text { Gemcitabine LD6H } \\
\qquad(\mathrm{n}=42)\end{array}$} & \multirow{2}{*}{$\begin{array}{c}\mathrm{P}^{\star} \text { Value for } \\
\text { Grade } \geq 3 \text { Toxicities }\end{array}$} \\
\hline & $\begin{array}{c}\text { All Grades } \\
\text { No (\%) }\end{array}$ & $\begin{array}{c}\text { Grade } \geq 3 \\
\text { No }(\%)\end{array}$ & $\begin{array}{c}\text { All Grades } \\
\text { No (\%) }\end{array}$ & $\begin{array}{c}\text { Grade } \geq 3 \\
\text { No }(\%)\end{array}$ & \\
\hline \multicolumn{6}{|l|}{ Non-Hematologic } \\
\hline ANOREXIA & $12(30)$ & $4(10)$ & $6(12.3)$ & $2(4.8)$ & 0.427 \\
\hline NAUSEA & $9(22.5)$ & $3(7.5)$ & $3(7.1)$ & 0 & 0.112 \\
\hline VOMITING & $8(20)$ & $3(7.5)$ & $6(14.3)$ & 0 & 0.112 \\
\hline DIARRHEA & $9(22.5)$ & $5(12.5)$ & $7(16.7)$ & $1(2.4)$ & 0.105 \\
\hline HYPOTENSION & $11(27.5)$ & $7(17.5)$ & $9(21.4)$ & $1(2.4)$ & 0.027 \\
\hline ALT & $9(22.5)$ & $5(12.5)$ & $9(21.4)$ & $2(4.8)$ & 0.259 \\
\hline AST & $10(25)$ & $5(12.5)$ & $6(14.3)$ & $2(4.8)$ & 0.259 \\
\hline CREATININE & $5(12.5)$ & $2(5)$ & $3(7.1)$ & 0 & 0.235 \\
\hline FATIGUE & $15(37.5)$ & $9(22.5)$ & $13(31)$ & $2(4.8)$ & 0.024 \\
\hline \multicolumn{6}{|l|}{ Hematologic } \\
\hline ANEMIA & $15(37.5)$ & $8(20)$ & $16(38.1)$ & $1(2.4)$ & 0.012 \\
\hline NEUTROPENIA & $16(40)$ & $9(22.5)$ & $9(21.4)$ & $1(2.4)$ & 0.006 \\
\hline THROMBOCYTOPENIA & $18(45)$ & $8(20)$ & $16(38.1)$ & $1(2.4)$ & 0.012 \\
\hline
\end{tabular}

Abbreviations: ALT, alanine aminotransferase; AST, aspartate transaminase; CI, confidence interval; CTCAE, the common terminology criteria for adverse events; LD6H, low dose over 6 hours; OR, odds ratio. *p value was calculated by the Ficher's exact test as all items having cell with expected count less than 5 .

Chi-square test was used as the comparative test between two arms regarding grade $3 / 4$ toxicity (Table 3 ). LD6H regimen had a significantly lower incidence of grade $3 / 4$ fatigue and hypotension when compared to the standard arm $(4.8 \%$ 


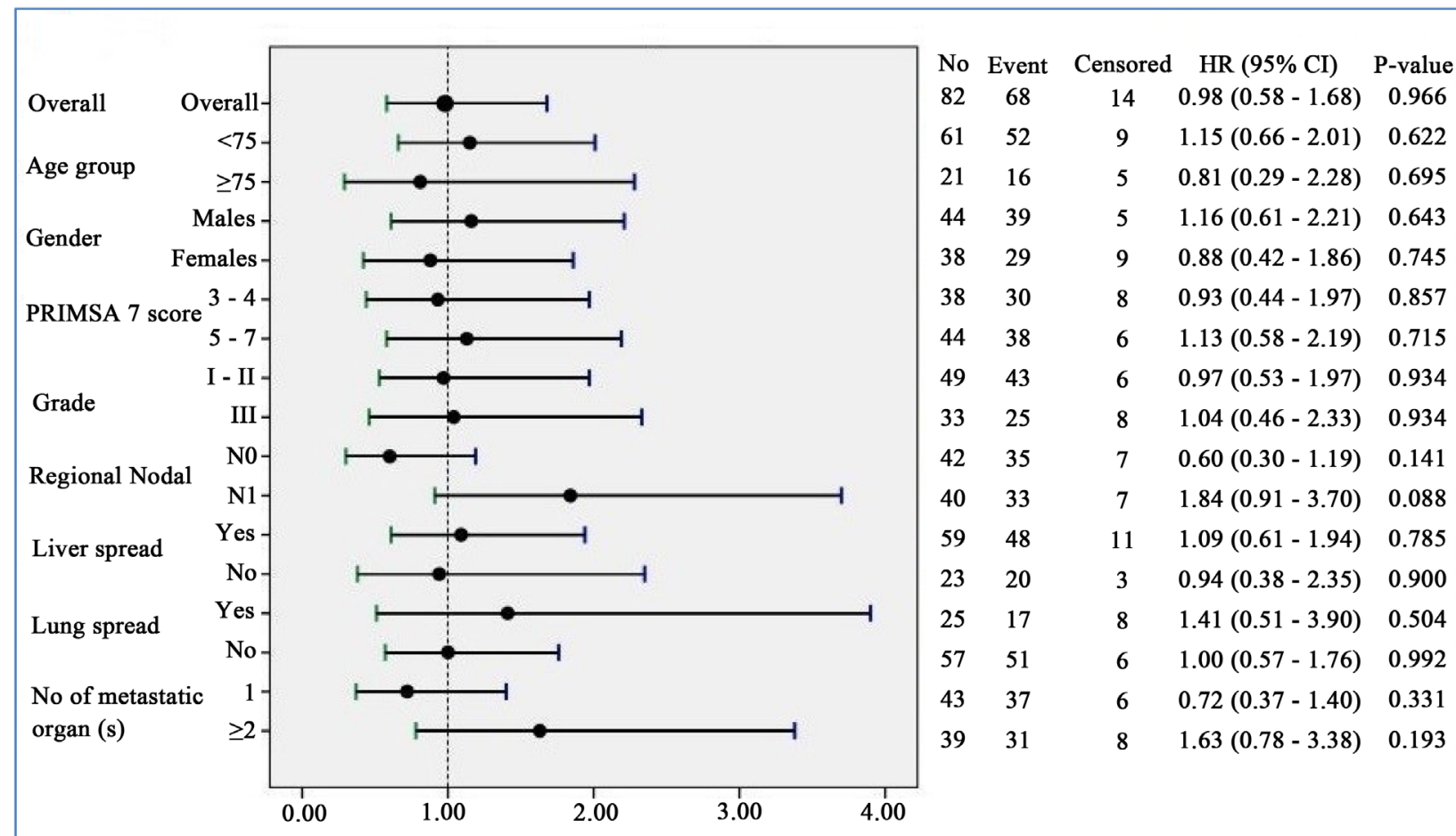

Favor of Low dose regimen Favor of Standard dose regimen

(a)

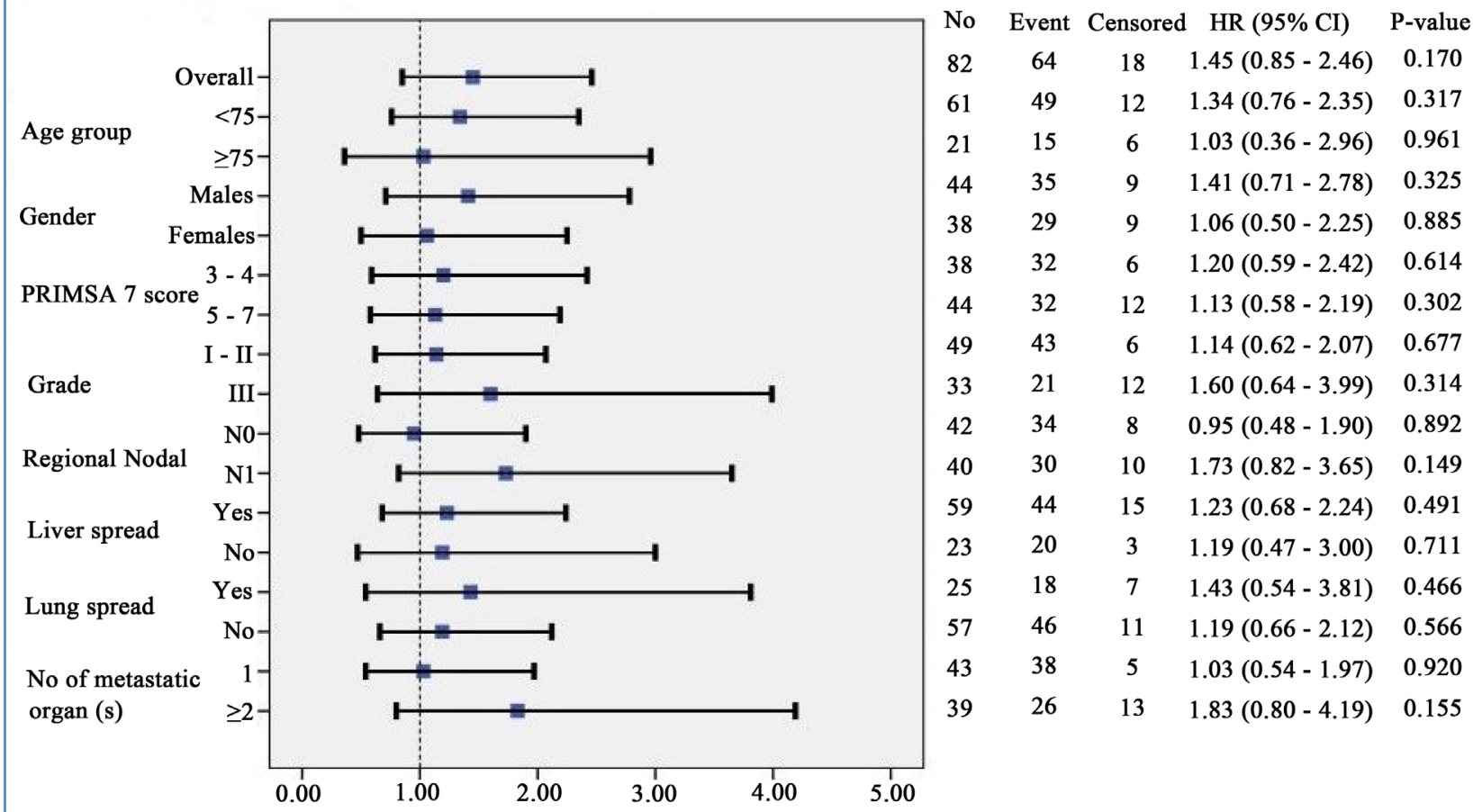

Favor Low dose regimen Favor Standard regimen

(b)

Figure 2. Prespecified subgroup analysis by forest plot, with HRs for (a) PFS and (b) OS. CI: confidence interval, HR: Hazard Ratio; OS: overall survival; PFS: progression free survival; PRIMSA: Program of Research to Integrate the Services for the Maintenance of Autonomy. 
vs. $22.5 \%$ respectively, $\mathrm{p}=0.024$ for fatigue, and $2.4 \%$ vs. $27.5 \%$ respectively, $\mathrm{p}=$ 0.027 for hypotension). For hematologic adverse events, there was a significantly lower incidence of grade 3/4 thrombocytopenia in $\mathrm{LD} 6 \mathrm{H}$ arm when compared to that in the standard protocol arm $(2.4 \%$ vs. $20 \%, \mathrm{p}=0.012)$. Grade $3 / 4$ neutropenia was much lower in $\mathrm{LD} 6 \mathrm{H}$ protocol than in the standard protocol $(2.4 \% \mathrm{vs}$. $22.5 \%$ respectively, $\mathrm{p}=0.006$ ). There were no treatment-related deaths. A $75 \%$ dose reduction was required in 4 patients in standard protocol arm, while no dose reduction was required for gemcitabine in patients received the low-dose regimen.

Our raw data is available in the attached commentary file 3.

\section{Discussion}

The treatment of metastatic pancreatic adenocarcinoma is slowly progressive in spite of its worst survival among all cancer [1]. The gemcitabine $1000 \mathrm{mg} / \mathrm{m}^{2}$ over 30-minute infusion on days 1, 8, and 15 of 4-week cycle became the standard regimen in patients with advanced or metastatic pancreatic cancer based on the randomized phase III study conducted by Burris et al. who proved a statistically significant survival advantage of gemcitabine arm when compared to 5-fluorouracil in term of median and 1-year OS [3]. Two large randomized studies documented the superiority of FOLFIRINOX regimen in PRODIGE 4/ACCORD 11 Phase III trial [4] and gemcitabine and nab-paclitaxel combination in MPACT phase III clinical trial [5] over gemcitabine alone. However, both trials did not take the factor of frailty into consideration. Also, in both trials, the age was not the parameter of patients' stratification (elderly patients and PS grade 2 were excluded from in PRODIGE 4/ACCORD 11 trial and insufficient data about outcome in relation to the age in MPACT trial).

To the best of our knowledge, our study is the first randomized phase II study comparing the standard gemcitabine protocol with low-dose gemcitabine over 6-hour infusion in frail elderly patients with advanced pancreatic cancer. In our trial, there was no significant difference between the standard group and LD6H group as regard ORR and DCR ( $17.5 \%$ vs. $21.4 \%$ respectively; $\mathrm{p}=0.654$ for ORR and $45 \%$ vs. $42.9 \%$ respectively; $\mathrm{p}=845)$. Also, there is no significant difference in median PFS, 5 months in both groups, $\mathrm{p}=0.908$; unadjusted HR 1.07; 95\% CI 0.60 - 1.90; adjusted HR 1.08; 95\% CI 0.65 - 1.7; hazard p = 0.762). Also, the result of OS is insignificant (median OS:10 months; 95\% CI 8.95 - 11.05 in standard arm versus 8 months, 95\% CI 6.41 - 11.59 in LD6H group; unadjusted HR 1.25 ; $95 \%$ CI 76 to $2.05 ; \mathrm{p}=0.331$ ). Subgroup analysis within the study arms revealed the absence of any significant effect of any subgroup of the examined parameters on PFS and OS. The low-dose regimen has a significantly lower incidence of adverse effects grades 3 or 4 when compared to the standard regimen: ( $4.8 \%$ vs. $22.5 \%$; $\mathrm{p}=0.024$ for fatigue, $2.4 \%$ vs. $27.5 \% ; \mathrm{p}=0.027$ for hypotension, $2.4 \%$ vs. $20 \% ; \mathrm{p}=0.012$ for each anemia and thrombocytopenia, $22.5 \%$ vs. $2.4 \%$, $\mathrm{p}=0.006$ for neutropenia). 
Our results agree with a phase II study conducted by Sakamoto et al. [21] who documented that low dose gemcitabine is less toxic and equally effective to the standard gemcitabine. However, this comparison is not dependable because of the presence of meaningful differences in patients and methods between the two studies as we used prolonged infusion time (6-hour) with frailty as eligible criterion and, the age and PRIMSA 7 score parameters as indicators of comparison, while they did not. The incidence of grade $3 / 4$ adverse events in the low-dose arm of our study is higher than that of the low-dose arm of Sakamoto study [21]. It may be due to the biological nature of our patients as the frail people have diminished the capacity to compensate for stressors compared to people of the same chronological age [10].

Matsumoto et al. [22] proved that the low-dose gemcitabine is superior to the best supportive care (BSC) in elderly (they do not report frailty.) In spite of using a low dose gemcitabine in elderly, we cannot compare our results with Matsumoto et al. [22], because the comparator arm is different (standard gemcitabine protocol in our study and BSC in Matsumoto study). Also, the dosage of the experimental arm is different in spite of named low-dose gemcitabine (250 $\mathrm{mg} / \mathrm{m}^{2}$ in our study and $600-800 \mathrm{mg} / \mathrm{m}^{2}$ in Matsumoto study).

The small sample size is one of the limitations of our study. Also, our sample size depends on availability of the eligible cases, not on the prior statistical calculation of that size.

\section{Conclusion}

Low-dose gemcitabine over 6-hour infusion is equally effective and less toxic when compared to standard gemcitabine protocol for frail elderly patients with advanced pancreatic adenocarcinoma. So, we recommend low-dose gemcitabine for frail elderly patients with advanced pancreatic cancer.

\section{Conflicts of Interest}

The authors declare no conflicts of interest regarding the publication of this paper.

\section{References}

[1] Bray, F., Ferlay, J., Soerjomataram, I., Siegel, R.L., Torre, L.A. and Jemal, A. (2018) Global Cancer Statistics 2018: GLOBOCAN Estimates of Incidence and Mortality Worldwide for 36 Cancers in 185 Countries. CA: A Cancer Journal for Clinicians, 68, 394-424. https://doi.org/10.3322/caac.21492

[2] Rawla, P., Sunkara, T. and Gaduputi, V. (2019) Epidemiology of Pancreatic Cancer: Global Trends, Etiology and Risk Factors. World Journal of Oncology, 10, 10-27. https://doi.org/10.14740/wjon1166

[3] Burris, H.A., Moore, M.J., Andersen, J., Green, M.R., Rothenberg, M.L., Modiano, M.R., et al. (1997) Improvements in Survival and Clinical Benefit with Gemcitabine as First-Line Therapy for Patients with Advanced Pancreas Cancer: A Randomized Trial. Journal of Clinical Oncology, 15, 2403-2413. https://doi.org/10.1200/JCO.1997.15.6.2403 
[4] Conroy, T., Desseigne, F., Ychou, M., Bouché, O., Guimbaud, R., Bécouarn, Y., et al. (2001) Groupe Tumeurs Digestives of Unicancer. PRODIGE Intergroup. FOLFIRINOX versus Gemcitabine for Metastatic Pancreatic Cancer. The New England Journal of Medicine, 364, 1817-1825.https://doi.org/10.1056/NEJMoa1011923

[5] Von Hoff, D.D., Ervin, T., Arena, F.P., Chiorean, E.G., Infante, J., Moore, M., et al. (2013) Increased Survival in Pancreatic Cancer with Nab-Paclitaxel plus Gemcitabine. The New England Journal of Medicine, 369, 1691-1703. https://doi.org/10.1056/NEJMoa1304369

[6] Macchini, M., Chiaravalli, M., Zanon, S., Peretti, U., Mazza, E., Gianni, L., et al. (2019) Chemotherapy in Elderly Patients with Pancreatic Cancer: Efficacy, Feasibility and Future Perspectives. Cancer Treatment Reviews, 72, 1-6.

https://doi.org/10.1016/j.ctrv.2018.10.013

[7] Rockwood, K., Song, X., MacKnight, C., Bergman, H., Hogan, D.B., McDowell, I., et al. (2005) A Global Clinical Measure of Fitness and Frailty in Elderly People. Canadian Medical Association Journal, 173, 489-495. https://doi.org/10.1503/cmaj.050051

[8] Turner, G. and Clegg, A. (2014) Best Practice Guidelines for the Management of Frailty: A British Geriatrics Society, Age UK and Royal College of General Practitioners Report. Age Ageing, 43, 744-747. https://doi.org/10.1093/ageing/afu138

[9] Raiche, M., Hebert, R. and Dubois, M.F. (2008) Prisma-7: A Case Finding Tool to Identify Older Adults with Moderate to Severe Disabilities. Archives of Gerontology and Geriatrics, 47, 9-18. https://doi.org/10.1016/j.archger.2007.06.004

[10] Hubbard, R.E., Peel, N.M., Samanta, M., Gray, L.C., Fries, B.E., Mitnitski, A. and Rockwood, K. (2015) Derivation of a Frailty Index from the Interrai Acute Care Instrument. BMC Geriatrics, 15, 27. https://doi.org/10.1186/s12877-015-0026-Z

[11] Orimo, H., Ito, H., Suzuki, T., Araki, A., Hosoi, T. and Sawabe, M. (2006) Reviewing the Definition of "Elderly". Geriatrics and Gerontology International, 6, 149-158. https://doi.org/10.1111/j.1447-0594.2006.00341.x

[12] Yamagishi, Y., Higuchi, H., Izumiya, M., Sakai, G., Iizuka, H., Nakamura, S., et al. (2010) Gemcitabine as First-Line Chemotherapy in Elderly Patients with Unresectable Pancreatic Carcinoma. Journal of Gastroenterology, 45, 1146-1154. https://doi.org/10.1007/s00535-010-0258-9

[13] Hentic, O., Dreyer, C., Rebours, V., Zappa, M., Levy, P., Raymond, E., et al. (2011) Gemcitabine in Elderly Patients with Advanced Pancreatic Cancer. World Journal of Gastroenterology, 17, 3497-3502. https://doi.org/10.3748/wjg.v17.i30.3497

[14] Higuera, O., Ghanem, I., Nasimi, R., Prieto, I., Koren, L. and Feliu, J. (2016) Management of Pancreatic Cancer in the Elderly. World Journal of Gastroenterology, 22, 764-775. https://doi.org/10.3748/wjg.v22.i2.764

[15] Ali, E.M., Maklad, A.M., Khallaf, S.M. and Samir, M.M. (2016) RM-029: Outcome of Continuous Infusion of Low Dose Gemcitabine in Locally Advanced and Metastatic Pancreatic Carcinoma. Annals of Oncology, 27, ii92. https://doi.org/10.1093/annonc/mdw201.28

[16] Schmid, P., Schweigert, M., Beinert, T., Flath, B., Sezer, O. and Possinger, K. (2005) Prolonged Infusion of Gemcitabine in Advanced Solid Tumors: A Phase-I-Study. Investigational New Drugs, 23, 139-146. https://doi.org/10.1007/s10637-005-5859-4

[17] Akrivakis, K., Schmid, P., Flath, B., Schweigert, M., Sezer, O. and Possinger, K. (1999) Prolonged Infusion of Gemcitabine in Stage IV Breast Cancer: A Phase I Study. Anticancer Drugs, 10, 525-532. https://doi.org/10.1097/00001813-199907000-00003 
[18] Eisenhauer, E.A., Therasse, P., Bogaerts, J., Schwartz, L.H., Sargent, D., Ford, R., et al. (2009) New Response Evaluation Criteria in Solid Tumours: Revised Recist Guideline (Version 1.1). European Journal of Cancer, 45, 228-247. https://doi.org/10.1016/j.ejca.2008.10.026

[19] Common Terminology Criteria for Adverse Events (CTCAE), Version 4.03 (2010) US Department of Health and Human Services. National Institutes of Health $\mathrm{Na}$ tional Cancer Institute.

http://evs.nci.nih.gov/ftp1/CTCAE/CTCAE_4.03_2010-06-14_QuickReference_5x7. pdf

[20] IBM Corp. Released. IBM SPSS Statistics for Windows 2012, V. 21.0. IBM, Armonk, NY.

[21] Sakamoto, H., Kitano, M., Suetomi, Y., Takeyama, Y., Ohyanagi, H., Nakai, T., et al. (2006) Comparison of Standard-Dose and Low-Dose Gemcitabine Regimens in Pancreatic Adenocarcinoma Patients: A Prospective Randomized Trial. Journal of Gastroenterology, 41, 70-76. https://doi.org/10.1007/s00535-005-1724-7

[22] Matsumoto, K., Miyake, Y., Kato, H., Kawamoto, H., Imagawa, A., Toyokawa, T., et al. (2011) Effect of Low-Dose Gemcitabine on Unresectable Pancreatic Cancer in Elderly Patients. Digestion, 84, 230-235. https://doi.org/10.1159/000330384 


\section{List of Abbreviations}

BSC: Best Supportive care.

CIs: Confidence Intervals.

CR: Complete Response.

CTCAE: Common Terminology Criteria for Adverse Events.

DCR: Disease Control Rate.

ECOG: Eastern Cooperative Oncology Group.

ERCP: Endoscopic Retrograde Cholangiopancreatography.

FDR: Fixed-Dose Rate.

HR: Hazard Ratio.

LD6H*: Low-Dose over 6-Hour.

OS: Overall Survival.

PFS: Progression Free Survival.

PRIMSA 7: Program of Research to Integrate the Services for the Maintenance of Autonomy.

PR: Partial Response.

PS: Performance Status.

RECIST: Response Evaluation Criteria in Solid Tumors.

SD: Stable Disease.

SPSS: Statistical Package for Social Sciences.

TUGT: Timed-Up-and-Go-Test.

Vs.: Versus.

WHO: World Health Organization.

${ }^{\star} \mathrm{LD} 6 \mathrm{H}$ is not standard abbreviation, but it is abbreviated as it appears more than three times in the text

\section{Appendix I: Consort Diagram}

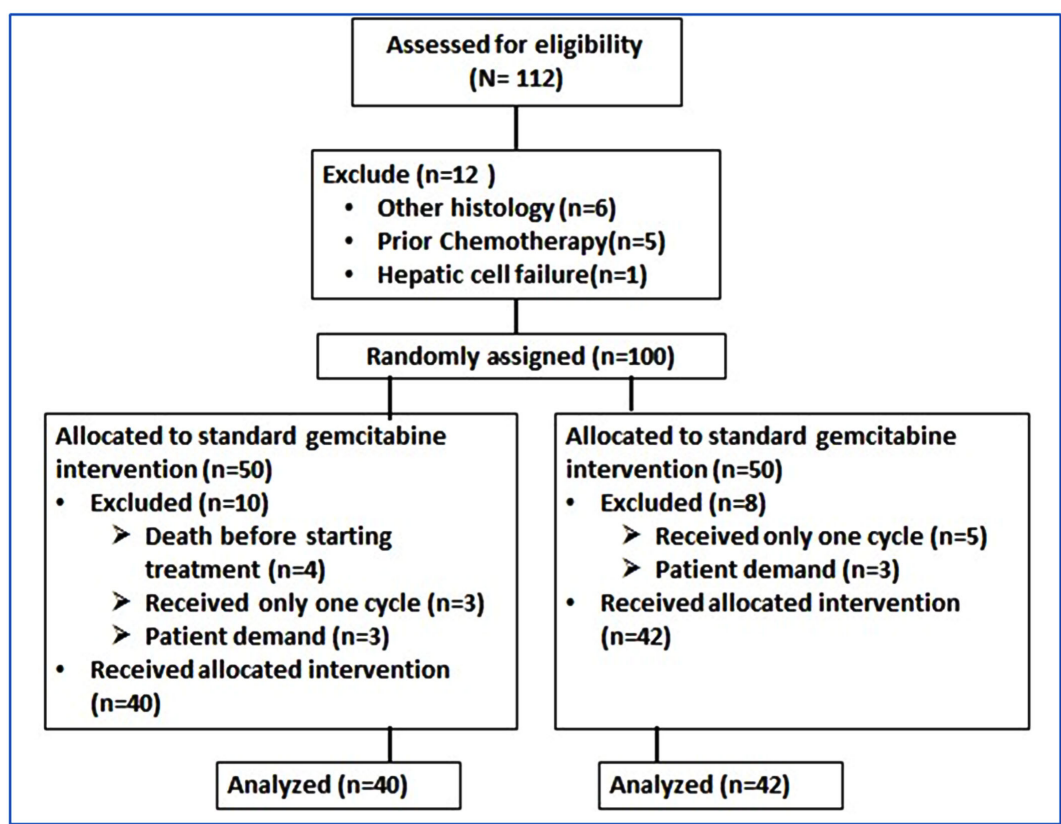




\section{Appendix II: Consort 2010 Checklist}

\section{CONSORT}

\section{Consort 2010 checklist of information to include when reporting a randomised trial*.}

\begin{tabular}{|c|c|c|c|}
\hline Section/Topic & Item No & Checklist item & Reported on page No \\
\hline \multicolumn{4}{|c|}{ Title and abstract } \\
\hline & 1a & Identification as a randomised trial in the title & P1:Title \\
\hline & $1 b$ & $\begin{array}{l}\text { Structured summary of trial design, methods, results, and } \\
\text { conclusions (for specific guidance see CONSORT for abstracts) }\end{array}$ & P1: Abstract \\
\hline \multicolumn{4}{|c|}{ Introduction } \\
\hline \multirow{2}{*}{$\begin{array}{l}\text { Background } \\
\text { and objectives }\end{array}$} & $2 \mathrm{a}$ & Scientific background and explanation of rationale & P2, P3: Background \\
\hline & $2 b$ & Specific objectives or hypotheses & P3: Background \\
\hline \multicolumn{4}{|c|}{ Methods } \\
\hline \multirow{2}{*}{ Trial design } & $3 a$ & $\begin{array}{l}\text { Description of trial design (such as parallel, factorial) } \\
\text { including allocation ratio }\end{array}$ & P5, Study design and treatment plan \\
\hline & $3 b$ & $\begin{array}{l}\text { Important changes to methods after trial commencement } \\
\text { (such as eligibility criteria), with reasons }\end{array}$ & P5: Study design and treatment plan \\
\hline \multirow{2}{*}{ Participants } & $4 a$ & Eligibility criteria for participants & P4: Patients selection \\
\hline & $4 \mathrm{~b}$ & Settings and locations where the data were collected & P4: Patients selection \\
\hline Interventions & 5 & $\begin{array}{l}\text { The interventions for each group with sufficient details to } \\
\text { allow replication, including how and when they } \\
\text { were actually administered }\end{array}$ & P5: Study design and treatment plan \\
\hline \multirow{2}{*}{ Outcomes } & $6 a$ & $\begin{array}{l}\text { Completely defined pre-specified primary and secondary } \\
\text { outcome measures, including how and when they were assessed }\end{array}$ & P6: Statistical Analysis \\
\hline & $6 \mathrm{~b}$ & $\begin{array}{l}\text { Any changes to trial outcomes after the trial commenced, with } \\
\text { reasons }\end{array}$ & NA \\
\hline \multirow[b]{2}{*}{ Sample size } & $7 a$ & How sample size was determined & P4: Sample size \\
\hline & $7 \mathrm{~b}$ & $\begin{array}{l}\text { When applicable, explanation of any interim } \\
\text { analyses and stopping guidelines }\end{array}$ & NA \\
\hline \multicolumn{4}{|l|}{ Randomisation: } \\
\hline \multirow{2}{*}{$\begin{array}{l}\text { Sequence } \\
\text { generation }\end{array}$} & $8 \mathrm{a}$ & Method used to generate the random allocation sequence & P5: Study design and treatment plan \\
\hline & $8 \mathrm{~b}$ & $\begin{array}{l}\text { Type of randomisation; details of any restriction } \\
\text { (such as blocking and block size) }\end{array}$ & P5: Study design and treatment plan \\
\hline $\begin{array}{l}\text { Allocation } \\
\text { concealment } \\
\text { mechanism }\end{array}$ & 9 & $\begin{array}{l}\text { Mechanism used to implement the random allocation } \\
\text { sequence (such as sequentially numbered containers), } \\
\text { describing any steps taken to conceal the sequence } \\
\text { until interventions were assigned }\end{array}$ & P5: Study design and treatment plan \\
\hline Implementation & 10 & $\begin{array}{l}\text { Who generated the random allocation sequence, who enrolled } \\
\text { participants, and who assigned participants to interventions }\end{array}$ & P5: Study design and treatment plan \\
\hline \multirow[t]{2}{*}{ Blinding } & $11 \mathrm{a}$ & $\begin{array}{l}\text { If done, who was blinded after assignment to interventions } \\
\text { (for example, participants, care providers, } \\
\text { those assessing outcomes) and how }\end{array}$ & NA \\
\hline & $11 \mathrm{~b}$ & If relevant, description of the similarity of interventions & NA \\
\hline
\end{tabular}


Statistical
$12 \mathrm{a}$

methods
Participant flow (a diagram is strongly recommended)

Recruitment

Baseline data

Numbers analysed

Outcomes and estimation

Ancillary analyses

Harms

Generalisability

Interpretation

Registration

Protocol

Funding

\section{randomly assigned, received intended treatment,}

For each group, the numbers of participants who wer and were analysed for the primary outcome

For each group, losses and exclusions after randomisation, together with reasons

Dates defining the periods of recruitment and follow-up

Why the trial ended or was stopped

A table showing baseline demographic and clinical characteristics for each group

For each group, number of participants (denominator) included in each analysis and whether the analysis was by original assigned groups

For each primary and secondary outcome, results for each group, and the estimated effect size and its precision (such as $95 \%$ confidence interval)

For binary outcomes, presentation of both absolute and relative effect sizes is recommended

Results of any other analyses performed, including subgroup analyses and adjusted analyses, distinguishing pre-specified from exploratory

All important harms or unintended effects in each group (for specific guidance see CONSORT for harms)

\section{Discussion}

Trial limitations, addressing sources of potential bias, imprecision, and, if relevant, multiplicity of analyses

Generalisability (external validity, applicability) of the trial findings

Interpretation consistent with results, balancing benefits and harms, and considering other relevant evidence

\section{Other information}

Registration number and name of trial registry

Where the full trial protocol can be accessed, if available

Sources of funding and other support (such as supply of drugs), role of funders
P6: Statistical Analysis

P7: Statistical Analysis

P7: Demographics and Patients' characteristics

P7: Losses and exclusions after randomisation patient's follow-up

P7: Losses and exclusions after randomisation patient's follow-up

P7: Losses and exclusions after randomisation patient's follow-up

Demographics and Patients' characteristics

Demographics and Patients' characteristics

P8: Survival end-points

NA

P8: Survival end-points

P8, P9: Adverse Events

NA

P10: Paragraph 2 and 3

P11: Paragraph 1

P10: Paragraph 2 and 3

P5: Paragraph 1

P5: Study design and treatment plan

NA

${ }^{*}$ We strongly recommend reading this statement in conjunction with the CONSORT 2010 Explanation and Elaboration for important clarifications on all the items. If relevant, we also recommend reading CONSORT extensions for cluster randomised trials, non-inferiority and equivalence trials, non-pharmacological treatments, herbal interventions, and pragmatic trials. Additional extensions are forthcoming: for those and for up to date references relevant to this checklist, see http://www.consort-statement.org/. 


\section{Appendix III: Frailty-Prisma 7}

\section{$B C$ Guidelines.ca \\ Guidelines \& Protocols Advisory Committee}

\section{PRISMA-7 Questionnaire}

\section{PATIENT Questions}

1. Are you older than 85 years?

Yes $\square \quad$ No $\square$

2. Are you male?

Yes $\square \quad$ No $\square$

3. In general, do you have any health problems that require you to limit your activities?

Yes $\square \quad$ No $\square$

4. Do you need someone to help you on a regular basis?

Yes $\square \quad$ No $\square$

5. In general, do you have any health problems that require you to stay at home?

Yes $\square \quad$ No $\square$

6. If you need help, can you count on someone close to you?

Yes $\square \quad$ No $\square$

7. Do you regularly use a stick, walker or wheelchair to move about?

Yes $\square$

No $\square$

Total checked:

\section{Instructions:}

- For questions 3 through 7, do not interpret the answer; simply note the person's answer without considering whether or not it should be "yes" or "no".

- If the respondent hesitates between "yes" and "no", ask him/her to choose one of the two answers.

- If, despite several attempts, he/she persists in answering "a little" or "at times", enter "yes".

SCORING: If the respondent had 3 or more "yes" answers, this indicates an increased risk of frailty and the need for further clinical review.

\section{Reference:}

Raîche, M., R. Hébert, M-F. Dubois, and the PRISMA partners. User guide for the PRISMA-7 questionnaire to identify elderly people with severe loss of autonomy. In Integrated service delivery to ensure persons' functional autonomy, ed. R. Hébert, A. Tourigny, and M. Gagnon, 14765. Quebec: Edisem.

PRISMA (Program of Research on Integration of Services for the Maintenance of Autonomy) is funded by the Canadian Health Services Research Foundation in partnership with the "Fonds de la Recherche en Santé du Québec". For more information, see www.prisma-qc.ca. 
Complementary File 1: Multivariate Analysis for PFS

\begin{tabular}{|c|c|c|c|c|c|c|c|c|}
\hline \multicolumn{9}{|c|}{ Variables in the Equation } \\
\hline \multirow{2}{*}{ Characteristics } & \multirow{2}{*}{ B } & \multirow{2}{*}{ SE } & \multirow{2}{*}{ Wald } & \multirow{2}{*}{ df } & \multirow{2}{*}{ Sig. } & \multirow{2}{*}{$\begin{array}{l}\text { Exp } \\
\text { (B) }\end{array}$} & \multicolumn{2}{|c|}{$95.0 \%$ CI for $\operatorname{Exp}(B)$} \\
\hline & & & & & & & Lower & Upper \\
\hline Chemotherapy type & 0.078 & 0.258 & 0.092 & 1 & 0.762 & 1.081 & 0.652 & 1.792 \\
\hline Age group & -0.241 & 0.329 & 0.534 & 1 & 0.465 & 0.786 & 0.412 & 1.499 \\
\hline Gender & 0.158 & 0.261 & 0.364 & 1 & 0.546 & 1.171 & 0.701 & 1.954 \\
\hline PRIMSA_7_Score & 0.556 & 0.166 & 11.213 & 1 & 0.001 & 1.744 & 1.259 & 2.415 \\
\hline Tumor site & 0.089 & 0.148 & 0.358 & 1 & 0.550 & 1.093 & 0.817 & 1.461 \\
\hline Tumor Grades & -0.116 & 0.142 & 0.673 & 1 & 0.412 & 0.890 & 0.674 & 1.175 \\
\hline Nodal stage & -0.078 & 0.331 & 0.056 & 1 & 0.813 & 0.925 & 0.483 & 1.770 \\
\hline Liver met & -0.040 & 0.364 & 0.012 & 1 & 0.913 & 0.961 & 0.471 & 1.960 \\
\hline Bone met & 0.466 & 0.464 & 1.010 & 1 & 0.315 & 1.594 & 0.642 & 3.957 \\
\hline Lung met & -0.421 & 0.354 & 1.415 & 1 & 0.234 & 0.656 & 0.328 & 1.314 \\
\hline Distal Lymph N met & 0.700 & 0.392 & 3.193 & 1 & 0.074 & 2.013 & 0.934 & 4.338 \\
\hline Number of Metastatic organ(s) & -0.114 & 0.443 & 0.066 & 1 & 0.797 & 0.893 & 0.375 & 2.126 \\
\hline
\end{tabular}

Complementary File 2: Multivariate Analysis for OS

\begin{tabular}{|c|c|c|c|c|c|c|c|c|}
\hline \multicolumn{9}{|c|}{ Variables in the Equation } \\
\hline & \multirow{2}{*}{ B } & \multirow{2}{*}{ SE } & \multirow{2}{*}{ Wald } & \multirow{2}{*}{$\mathrm{df}$} & \multirow{2}{*}{ Sig. } & \multirow{2}{*}{$\begin{array}{l}\text { Exp } \\
\text { (B) }\end{array}$} & \multicolumn{2}{|c|}{$95.0 \%$ CI for $\operatorname{Exp}(\mathrm{B})$} \\
\hline & & & & & & & Lower & Upper \\
\hline CTRtype & 0.370 & 0.270 & 1.879 & 1 & 0.170 & 1.448 & 0.853 & 2.460 \\
\hline Age_group & -0.175 & 0.356 & 0.242 & 1 & 0.623 & 0.839 & 0.418 & 1.686 \\
\hline $\operatorname{sex}$ & -0.494 & 0.270 & 3.351 & 1 & 0.067 & 0.610 & 0.360 & 1.036 \\
\hline PRIMSA_7_Score & 0.357 & 0.157 & 5.174 & 1 & 0.023 & 1.430 & 1.051 & 1.945 \\
\hline Tsite & 0.242 & 0.141 & 2.943 & 1 & 0.086 & 1.274 & 0.966 & 1.681 \\
\hline Grades & -0.052 & 0.151 & 0.120 & 1 & 0.729 & 0.949 & 0.706 & 1.275 \\
\hline $\mathrm{N}$ & -0.078 & 0.338 & 0.053 & 1 & 0.817 & 0.925 & 0.477 & 1.795 \\
\hline livermet & 0.089 & 0.375 & 0.056 & 1 & 0.812 & 1.093 & 0.524 & 2.280 \\
\hline bonemet & 0.830 & 0.501 & 2.740 & 1 & 0.098 & 2.293 & 0.858 & 6.128 \\
\hline lungmet & 0.092 & 0.365 & 0.064 & 1 & 0.800 & 1.097 & 0.537 & 2.242 \\
\hline LNmet & 0.658 & 0.424 & 2.407 & 1 & 0.121 & 1.932 & 0.841 & 4.437 \\
\hline No_Met_Organ & -0.166 & 0.503 & 0.110 & 1 & 0.741 & 0.847 & 0.316 & 2.267 \\
\hline
\end{tabular}

